\title{
Elastic Buckling Behaviour of a Four-Lobed Cross Section Cylindrical Shell with Variable Thickness under Non-Uniform Axial Loads
}

\author{
Mousa Khalifa Ahmed \\ Department of Mathematics, Faculty of Science at Qena, South Valley University, 83523 Qena, Egypt
}

Correspondence should be addressed to Mousa Khalifa Ahmed, mousa@japan.com

Received 10 June 2009; Revised 5 October 2009; Accepted 28 October 2009

Recommended by Carlo Cattani

The static buckling of a cylindrical shell of a four-lobed cross section of variable thickness subjected to non-uniform circumferentially compressive loads is investigated based on the thin-shell theory. Modal displacements of the shell can be described by trigonometric functions, and Fourier's approach is used to separate the variables. The governing equations of the shell are reduced to eight first-order differential equations with variable coefficients in the circumferential coordinate, and by using the transfer matrix of the shell, these equations can be written in a matrix differential equation. The transfer matrix is derived from the nonlinear differential equations of the cylindrical shells by introducing the trigonometric series in the longitudinal direction and applying a numerical integration in the circumferential direction. The transfer matrix approach is used to get the critical buckling loads and the buckling deformations for symmetrical and antisymmetrical shells. Computed results indicate the sensitivity of the critical loads and corresponding buckling modes to the thickness variation of cross section and the radius variation at lobed corners of the shell.

Copyright (C) 2009 Mousa Khalifa Ahmed. This is an open access article distributed under the Creative Commons Attribution License, which permits unrestricted use, distribution, and reproduction in any medium, provided the original work is properly cited.

\section{Introduction}

The use of cylindrical shells which have noncircular profiles is common in many fields, such as aerospace, mechanical, civil and marine engineering structures. The displacement buckling modes of thin elastic shells essentially depend on some determining functions such as the radius of the curvature of the neutral surface, the shell thickness, the shape of the shell edges, and so forth. In simple cases when these functions are constant, the buckling modes occupy the entire shell surface. If the determining functions vary from point to point of the neutral surface then localization of the displacement buckling modes lies near the weakest lines on the shell surface, and this kind of problems is too difficult because the radius of its curvature varies with the circumferential coordinate, closed-form or analytic solutions cannot be obtained, in general, for this class of shells, numerical or approximate 
techniques are necessary for their analysis. Buckling has become more of a problem in recent years since the use of high-strength material requires less material for load support-structures and components have become generally more slender and buckle-prone. Many researchers have considerable interest in the study of stability problems of circular cylindrical shells under uniform axial loads with constant thickness and numerous investigations have been devoted to this, for example [1-9]. Other related references may be found in the well-known work of Love [10], Flügge [11] and Tovstik [12]. In contrast, the buckling behaviour under applied non-uniform axial loads has received much less attention, but some of treatments are found in [13-17], and Song [18] provided a review of research and trends in the area of stability of unstiffened circular cylindrical shells under non-uniform axial loads. Recently, with the advent of the high-speed digital computer, the study of vibration and buckling for shells directed to ones with complex geometry, such as the variability of radius of curvature and thickness. Using the modified Donell-type stability equations of cylindrical shells with applying Galerkin's method, the stability of cylindrical shells with variable thickness under dynamic external pressure is studied by Sofiyev and Erdem [19]. Eliseeva and Filippov [20], and Filippov et al. [21] presented the vibration and buckling of cylindrical shells of variable thickness with slanted and curvelinear edges, respectively, using the asymptotic and finite element methods. The analytical solutions for axisymmetric transverse vibration of cylindrical shells with thickness varying in power form due to forces acting in the transverse direction are derived for the first time by Duan and Koh [22]. Sambandam et al. [23] studied the buckling characteristics of cross-ply elliptical cylindrical shells under uniform axial loads based on the higher-order theory and found that an increase in the value of radius-tothickness ratio causes the critical load to decrease. Using the generalized beam theory, the influence of member length on the critical loads of elliptical cylindrical shells under uniform compression is studied by Silvestre [24]. A treatise on the use of the transfer matrix approach for mechanical science problems is presented by Tesar and Fillo [25]. However, the problem of stability of the shell-type structures treated here which are composed of circular cylindrical panels and flat plates with circumferential variable thickness under non-uniform loads does not appear to have been dealt with in the literature. The aim of this paper is to present the buckling behaviour of an isotropic cylindrical shell with a four-lobed cross section of circumferentially varying thickness, subjected to non-uniformly compressive loads, using the transfer matrix method and modeled on the thin-shell theory. The transfer matrix is derived from the nonlinear differential equations system for the cylindrical shell by numerical integration. The method is applied to symmetrical and antisymmetrical shells. The critical buckling loads and corresponding buckling deformations of the shell are presented. The influences of the thickness variation and radius variation on the buckling characteristics are examined. The results are cited in tabular and graphical forms.

\section{Theory and Formulation of the Problem}

It has been mentioned in Section 1 that the problem structure is modeled by thin-shell theory. In order to have a better representation, the shell geometry and governing equations are modeled as separate parts. The formulation of these parts is presented below.

\subsection{Geometrical Formulation}

We consider an isotropic, elastic, cylindrical shell of a four-lobed cross section profile expressed by the equation $r=a f(\theta)$, where $r$ is the varied radius along the cross section 
midline, $a$ is the reference radius of curvature, chosen to be the radius of a circle having the same circumference as the four-lobed profile, and $f(\theta)$ is a prescribed function of $\theta$ and can be described as

$$
\begin{gathered}
f(\theta)=a_{1}\left\{\begin{array}{cc}
\sec \theta, \quad \rho=0, & 0<\theta<\theta_{1} \\
(1-\zeta)(\sin \theta+\cos \theta)+\sqrt{\zeta^{2}-(1-\zeta)^{2}(1-2 \sin \theta \cos \theta)}, & \theta_{1}<\theta<90^{0}-\theta_{1} \\
\operatorname{cosec} \theta, \quad \rho=0, & 90^{0}-\theta_{1}<\theta<90^{0}
\end{array}\right\}, \\
a_{1}=\frac{A_{1}}{a}, \quad \zeta=\frac{R_{1}}{A_{1}}, \quad \theta_{1}=\tan ^{-1}(1-\zeta) .
\end{gathered}
$$

$L_{1}$ and $L_{2}$ are the axial and circumferential lengths of the middle surface of the shell, and the thickness $H(\theta)$ is varying continuously in the circumferential direction. The cylindrical coordinates $(x, s, z)$ are taken to define the position of a point on the middle surface of the shell, as shown in Figure 1(a), and Figure 1(b) shows the four-lobed cross section profile of the middle surface, with the apothem denoted by $A_{1}$, and the radius of curvature at the lobed corners by $R_{1}$. While $u, v$ and $w$ are the deflection displacements of the middle surface of the shell in the longitudinal, circumferential and transverse directions, respectively. We suppose that the shell thickness $H$ at any point along the circumference is small and depends on the coordinate $\theta$ and takes the following form:

$$
H(\theta)=h_{0} \varphi(\theta),
$$

where $h_{0}$ is a small parameter, chosen to be the average thickness of the shell over the length $L_{2}$. For the cylindrical shell which its cross section is obtained by the cutaway the circle of the radius $r_{0}$ from the circle of the radius $R_{0}$ (see Figure $1(\mathrm{c})$ ) function $\varphi(\theta)$ has the form: $\varphi(\theta)=1+\delta(1-\cos \theta)$, where $\delta$ is the amplitude of thickness variation, $\delta=d / h_{0}$, and $d$ is the distance between the circles centers. In general case $h_{0}=H(\theta=0)$ is the minimum value of $\varphi(\theta)$ while $h_{m}=H(\theta=\pi)$ is the maximum value of $\varphi(\theta)$, and in case of $d=0$ the shell has constant thickness $h_{0}$. The dependence of the shell thickness ratio $\eta=h_{m} / h_{0}$ on $\delta$ has the form $\eta=1+2 \delta$.

\subsection{Governing Equations}

For a general circular cylindrical shell subjected to a non-uniform circumferentially compressive load $p(\theta)$, the static equilibrium equations of forces, based on the GoldenveizerNovozhilov theory $[26,27]$, can be shown to be of the following forms:

$$
\begin{gathered}
N_{x}^{\prime}+N_{s x}^{\bullet}-P(\theta) u^{\prime \prime}=0, \quad N_{x s}^{\prime}+N_{s}^{\bullet}+\frac{Q_{s}}{R}-P(\theta) v^{\prime \prime}=0, \\
Q_{x}^{\prime}+Q_{s}^{\bullet}-\frac{N_{s}}{R}-P(\theta) w^{\prime \prime}=0, \quad M_{x}^{\prime}+M_{s x}^{\bullet}-Q_{x}=0, \\
M_{x s}^{\prime}+M_{s}^{\bullet}-Q_{s}=0, \quad S_{s}-Q_{s}-M_{s x}^{\prime}=0, \quad N_{x s}-N_{s x}-\frac{M_{s x}}{R}=0,
\end{gathered}
$$




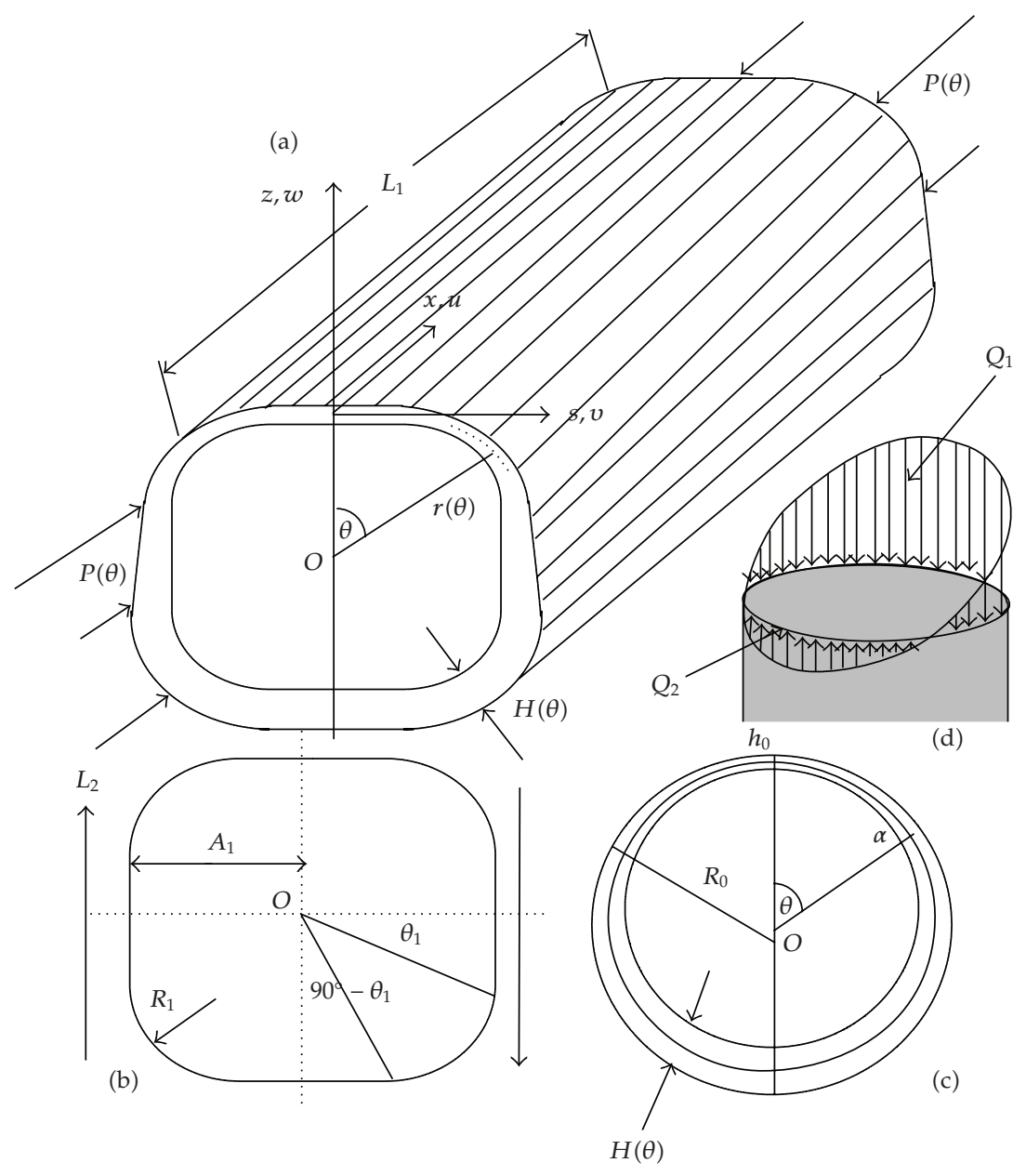

Figure 1: Coordinate system and geometry of a variable axial loaded cylindrical shell of four-lobed cross section with circumferential variable thickness.

where $N_{x}, N_{s}$ and $Q_{x}, Q_{s}$ are the normal and transverse shearing forces in the $x$ and $s$ directions, respectively, $N_{s x}$ and $N_{x s}$ are the in-plane shearing forces, $M_{x}, M_{s}$ and $M_{x s}, M_{s x}$ are the bending moment and the twisting moment, respectively, $S_{s}$ is the equivalent (Kelvin-Kirchoff) shearing force, $R$ is the radius of curvature of the middle surface, $' \equiv \partial / \partial x$, and ${ }^{\bullet} \equiv \partial / \partial s$. We assume that the shell is loaded along the circumferential coordinate with non-uniform axial loads $p(\theta)$ which vary with $\theta$, where the compressive load does not reach its critical value at which the shell loses stability. Generally, the form of the non-uniform load may be expressed as:

$$
p(\theta)=p_{0} g(\theta)
$$

where $g(\theta)$ is a given function of $\theta$ and $p_{0}$ is a constant. We assume that the shell is loaded by axially non-uniform loads $P(\theta)$ and takes the form as in [13]:

$$
p(\theta)=p_{0}(1+2 \cos \theta), \quad g(\theta)=1+2 \cos \theta
$$


and the sketch depicting this load is given in Figure 1(d). The applied load in this form establishes two zones on the shell surface: one is the compressive zone, $Q_{1}$, for $(0<\theta<2 \pi / 3)$ where the buckling load factor is a maximum and the thickness is a minimum and the other is the tensile zone, $Q_{2}$, for $(2 \pi / 3<\theta<\pi)$ where the buckling load factor is a minimum and the thickness is a maximum, as shown in this figure. Note that $p(\theta)=p_{0}$ in the case of applied uniform axial loads. Hereby, we deduce the following ratio of critical loads:

$$
\mu=\frac{p_{C} \text { for uniform load }}{p_{C} \text { for non-uniform load }}
$$

$p_{C}$ is the lowest value of applied compressive loads and named by the critical load.

The relations between strains and deflections for the cylindrical shells used here are taken from [28] as follows:

$$
\begin{gathered}
\varepsilon_{x}=u^{\prime}, \quad \varepsilon_{s}=v^{\bullet}+\frac{w}{R}, \quad \gamma_{x s}=v^{\prime}+u^{\bullet}, \quad \gamma_{x z}=w^{\prime}+\psi_{x}=0, \\
\gamma_{s z}=w^{\bullet}+\psi_{s}-\frac{v}{R}=0, \quad k_{x}=\psi_{x}^{\prime}, \quad k_{s}=\psi_{s}^{\bullet}+\frac{v^{\bullet}+w / R}{R}, \quad k_{s x}=\psi_{s}^{\prime}, \quad k_{x s}=\psi_{x}^{\bullet}+\frac{v^{\prime}}{R},
\end{gathered}
$$

where $\varepsilon_{x}$ and $\varepsilon_{s}$ are the normal strains of the middle surface of the shell, $\gamma_{x s}, \gamma_{x z}$ and $\gamma_{s z}$ are the shear strains, and the quantities $k_{x}, k_{s}, k_{s x}$ and $k_{x s}$ representing the change of curvature and the twist of the middle surface, $\psi_{x}$ is the bending slope, and $\psi_{s}$ is the angular rotation. The components of force and moment resultants in terms of (2.8) are given as:

$$
\begin{aligned}
& N_{x}=D\left(\varepsilon_{x}+v \varepsilon_{s}\right), \quad N_{s}=D\left(\varepsilon_{s}+v \varepsilon_{x}\right), \quad N_{x s}=\frac{D(1-v) \gamma_{x s}}{2}, \\
& M_{x}=K\left(k_{x}+v k_{s}\right), \quad M_{s}=K\left(k_{s}+v k_{x}\right), \quad M_{s x}=k(1-v) k_{s x} .
\end{aligned}
$$

From (2.4)-(2.9), with eliminating the variables $Q_{x}, Q_{s}, N_{x}, N_{x s}, M_{x}, M_{x s}$ and $M_{s x}$ which are not differentiated with respect to $s$, the system of the partial differential equations for the state variables $u, v, w, \psi_{s}, M_{s}, S_{s}, N_{s}$ and $N_{s x}$ of the shell is obtained as follows:

$$
\begin{gathered}
u^{\bullet}=\frac{2}{D(1-v) N_{s x}}+\left(\frac{H^{2}}{6 R}\right) \psi_{s}^{\prime}-v^{\prime}, \quad v^{\bullet}=\frac{N_{s}}{D}-\frac{w}{R}-v u^{\prime}, \quad w^{\bullet}=\frac{v}{r}-\psi_{s}, \\
\psi_{s}^{\bullet}=\frac{M_{s}}{K}+v \psi^{\prime}{ }_{x}-\frac{N_{s}}{R D}-\left(\frac{v}{R}\right) u^{\prime}, \quad M_{s}^{\bullet}=S_{s}-2 K(1-v) \psi^{\prime \prime}{ }_{s^{\prime}} \\
S_{s}^{\bullet}=\frac{N_{s}}{R}-v M_{s}^{\prime \prime}+K\left(1-v^{2}\right) w^{\prime \prime \prime}+P(\theta) w^{\prime \prime}, \quad N_{s}^{\bullet}=P(\theta) v^{\prime \prime}-\frac{S_{s}}{R}-N_{s x}^{\prime}, \\
N_{s x}^{\bullet}=D\left(1-v^{2}\right) u^{\prime \prime}+P(\theta) u^{\prime \prime}-v N_{s}^{\prime} .
\end{gathered}
$$

The quantities $D$ and $K$, respectively, are the extensional and flexural rigidities expressed in terms of the Young's modulus E, Poisson's ratio $v$ and the wall thickness $H(\theta)$ as the form: 
$D=E H /\left(1-v^{2}\right)$ and $K=E H^{3} / 12\left(1-v^{2}\right)$, and on considering the variable thickness of the shell, using (2.3), they take the form:

$$
\begin{gathered}
D=\left(\frac{E h_{0}}{1-v^{2}}\right) \varphi(\theta)=D_{0} \varphi(\theta), \\
K=\left(\frac{E\left(h_{0}\right)^{3}}{1-v^{2}}\right) \varphi^{3}(\theta)=K_{0} \varphi^{3}(\theta),
\end{gathered}
$$

where $D_{0}$ and $K_{0}$ are the reference extensional and flexural rigidities of the shell, chosen to be the averages on the middle surface of the shell over the length $L_{2}$.

For a simply supported shell, the solution of the system of (2.10) is sought as follows:

$$
\begin{gathered}
u(x, s)=\bar{U}(s) \cos \beta x, \quad(v(x, s), w(x, s))=(\bar{V}(s), \bar{W}(s)) \sin \beta x, \quad \psi_{s}(x, s)=\bar{\psi}_{s}(s) \sin \beta x \\
\left(N_{x}(x, s), N_{s}(x, s), Q_{s}(x, s), S_{s}(x, s)\right)=\left(\bar{N}_{x}(s), \bar{N}_{s}(s), \bar{Q}_{s}(s), \bar{S}_{s}(s)\right) \sin \beta x \\
\left(N_{x s}(x, s), N_{s x}(x, s), Q_{x}(x, s)\right)=\left(\bar{N}_{x s}(s), \bar{N}_{s x}(s), \bar{Q}_{x}(s)\right) \cos \beta x \\
\left(M_{x}(x, s), M_{s}(x, s)\right)=\left(\bar{M}_{x}(s), \bar{M}_{s}(s)\right) \sin \beta x \\
\left(M_{x s}(x, s), M_{s x}(x, s)\right)=\left(\bar{M}_{x s}(s), \bar{M}_{s x}(s)\right) \cos \beta x, \quad \beta=\frac{m \pi}{L_{1}}, m=1,2, \ldots
\end{gathered}
$$

where $m$ is the axial half-wave number, and the quantities $\bar{U}(s), \bar{V}(s), \ldots$ are the state variables and undetermined functions of $s$.

\section{Matrix Form of the Governing Equations}

The differential equations as shown previously are modified to a suitable form and solved numerically. Hence, by substituting (2.12) into (2.10), after appropriate algebraic operations and taking relations (2.11) into account, the system of buckling equations of the shell can be written in nonlinear ordinary differential equations referred to the variable $s$ only are obtained, in the following matrix form:

$$
a \frac{d}{d s}\left\{\begin{array}{c}
\tilde{U} \\
\tilde{V} \\
\widetilde{W} \\
\widetilde{\Psi}_{s} \\
\widetilde{M}_{s} \\
\widetilde{S}_{s} \\
\widetilde{N}_{s} \\
\widetilde{N}_{s x}
\end{array}\right\}=\left[\begin{array}{cccccccc}
0 & V_{12} & 0 & V_{14} & 0 & 0 & 0 & V_{18} \\
V_{21} & 0 & V_{23} & 0 & 0 & 0 & V_{27} & 0 \\
0 & V_{32} & 0 & V_{34} & 0 & 0 & 0 & 0 \\
V_{41} & 0 & V_{43} & 0 & V_{45} & 0 & V_{47} & 0 \\
0 & 0 & 0 & V_{54} & 0 & V_{56} & 0 & 0 \\
0 & 0 & V_{63} & 0 & V_{65} & 0 & V_{67} & 0 \\
0 & V_{72} & 0 & 0 & 0 & V_{76} & 0 & V_{78} \\
V_{81} & 0 & 0 & 0 & 0 & 0 & V_{87} & 0
\end{array}\right]\left\{\begin{array}{c}
\tilde{U} \\
\tilde{V} \\
\widetilde{W} \\
\widetilde{\Psi}_{s} \\
\widetilde{M}_{s} \\
\widetilde{S}_{s} \\
\widetilde{N}_{s} \\
\widetilde{N}_{s x}
\end{array}\right\} .
$$


By using the state vector of fundamental unknowns $Z(s)$, system (3.1) can be written as:

$$
\begin{aligned}
\left(a \frac{d}{d s}\right)\{Z(s)\} & =[V(s)]\{Z(s)\}, \\
\{Z(s)\} & =\left\{\tilde{U}, \widetilde{V}, \widetilde{W}, \widetilde{\psi}_{s}, \widetilde{M}_{s}, \widetilde{S}_{s}, \widetilde{N}_{s}, \widetilde{N}_{s x}\right\}^{T}, \\
(\tilde{U}, \tilde{V}, \widetilde{W}) & =k_{0}(\bar{U}, \bar{V}, \bar{W}), \quad \widetilde{\psi}_{s}=\left(\frac{k_{0}}{\beta}\right) \bar{\psi}_{s}, \quad \widetilde{M}_{s}=\left(\frac{1}{\beta^{2}}\right) \bar{M}_{s}, \\
\left(\widetilde{S}_{s}, \widetilde{N}_{s}, \widetilde{N}_{s x}\right) & =\left(\frac{1}{\beta^{3}}\right)\left(\bar{S}_{s}, \bar{N}_{s}, \bar{N}_{s x}\right) .
\end{aligned}
$$

For the noncircular cylindrical shell which cross section profile is obtained by function $(r=$ $a f(\theta)$ ), the hypotenuse $(d s)$ of a right triangle whose sides are infinitesimal distances along the surface coordinates of the shell takes the following form: $(d s)^{2}=(d r)^{2}+(r d \theta)^{2}$, then we have

$$
\frac{d s}{a}=\sqrt{(f(\theta))^{2}+\left(\frac{d f(\theta)}{d \theta}\right)^{2}} d \theta .
$$

Using (3.4), the system of buckling equations (3.2) takes the following form:

$$
\left(\frac{d}{d \theta}\right)\{Z(\theta)\}=\Psi(\theta)[V(\theta)]\{Z(\theta)\}
$$

where $\Psi(\theta)=\sqrt{(f(\theta))^{2}+(d f(\theta) / d \theta)^{2}}$, and the coefficients matrix $[V(\theta)]$ are given as:

$$
\begin{aligned}
& V_{12}=-\left(\frac{m \pi}{l}\right), \quad V_{14}=\left(\frac{m \pi}{l}\right)^{2}\left(\frac{h^{2}}{6}\right) \varphi, \quad V_{18}=\left(\frac{m \pi}{l}\right)^{2}\left(\frac{h^{2}}{6}(1-v) \varphi\right), \quad V_{21}=v\left(\frac{m \pi}{l}\right), \\
& V_{23}=-\rho, \quad V_{27}=\left(\frac{m \pi}{l}\right)^{3}\left(\frac{h^{2}}{12 \varphi}\right), \quad V_{32}=\rho, \quad V_{34}=-\left(\frac{m \pi}{l}\right), \quad V_{41}=-v \rho, \\
& V_{43}=-v\left(\frac{m \pi}{l}\right)^{2}, \quad V_{45}=\frac{1}{h \varphi^{3}}, \quad V_{46}=\frac{\rho h}{12 \varphi^{2}}, \quad V_{54}=2(1-v) h\left(\frac{m \pi}{l}\right)^{2} \varphi^{2}, \\
& V_{56}=1, \quad V_{63}=\frac{\left(1-v^{2}\right)(m \pi / l)^{4} \varphi^{3}}{2}-\frac{\bar{p} g}{(m \pi / l)}, \quad V_{65}=v\left(\frac{m \pi}{l}\right), \quad V_{67}=\rho\left(\frac{m \pi}{l}\right), \\
& V_{72}=-\frac{\bar{p} g}{(m \pi / l)}, \quad V_{76}=-\rho, \quad V_{78}=\frac{m \pi}{l}, \quad V_{81}=\frac{\varphi\left(1-v^{2}\right)\left(12 / h^{2}\right)}{m \pi l}-\frac{\bar{p} g}{(m \pi / l)},
\end{aligned}
$$

$V_{87}=-v(m \pi / l)$ in terms of the following dimensionless shell parameters: curvature 
parameter $\rho=a / R$, buckling load factor $\bar{p}=p_{0}\left(a^{2} / K_{0}\right), l=L_{1} / a$, and $h=h_{0} / a$. The state vector $\{Z(\theta)\}$ of fundamental unknowns can be easily expressed as:

$$
\{Z(\theta)\}=[Y(\theta)]\{Z(0)\},
$$

by using the transfer matrix $[Y(\theta)]$ of the shell, the substitution of the expression into (3.5) yields:

$$
\begin{gathered}
\left(\frac{d}{d \theta}\right)[Y(\theta)]=\Psi(\theta)[V(\theta)][Y(\theta)], \\
{[Y(0)]=[I] .}
\end{gathered}
$$

The governing system of buckling (3.8) is too complicated to obtain any closed-form solution, and this problem is highly favorable for solving by numerical methods. Hence, the matrix $[Y(\theta)]$ is obtained by using numerical integration, by use of the Runge-kutta integration method of forth-order, with the starting value $[Y(0)]=[I]$ (unit matrix) which is given by taking $\theta=0$ in (3.7), and its solution depends only on the geometric and martial properties of the shell. For a plane passing through the central axis in a shell with structural symmetry, symmetrical and antisymmetrical profiles can be obtained, and consequently, only one-half of the shell circumference is considered with the boundary conditions at the ends taken to be the symmetric or antisymmetric type of buckling deformations. Therefore, the boundary conditions for symmetrical and antisymmetrical bucking deformations are

$$
\tilde{V}=\widetilde{\psi}_{s}=0, \widetilde{S}_{s} \widetilde{N}_{s x}=0, \quad \tilde{U}=\widetilde{W}=0, \quad \widetilde{N}_{s}=\widetilde{M}_{s}=0, \quad \text { respectively }
$$

\section{Buckling Loads and Buckling Modes}

The substitution of (3.9) into (3.7) results in the following buckling equations:

$$
\begin{aligned}
& {\left[\begin{array}{llll}
Y_{21} & Y_{23} & Y_{25} & Y_{27} \\
Y_{41} & Y_{43} & Y_{45} & Y_{47} \\
Y_{61} & Y_{63} & Y_{65} & Y_{67} \\
Y_{81} & Y_{83} & Y_{85} & Y_{87}
\end{array}\right]_{(\pi)}\left\{\begin{array}{c}
\tilde{U} \\
\widetilde{W} \\
\widetilde{M}_{s} \\
\widetilde{N}_{s}
\end{array}\right\}_{(0)}=0, \text { for symmetrical modes, }} \\
& {\left[\begin{array}{llll}
Y_{12} & Y_{14} & Y_{16} & Y_{18} \\
Y_{32} & Y_{34} & Y_{36} & Y_{38} \\
Y_{52} & Y_{45} & Y_{56} & Y_{58} \\
Y_{72} & Y_{74} & Y_{76} & Y_{78}
\end{array}\right]_{(\pi)}\left\{\begin{array}{c}
\tilde{V} \\
\widetilde{\Psi}_{s} \\
\widetilde{S}_{s} \\
\widetilde{N}_{s x}
\end{array}\right\}_{(0)}=0, \text { for antisymmetrical modes. }}
\end{aligned}
$$


The matrices $[Y(\pi)]$ depend on the buckling load factor $\bar{p}$ and the circumferential angle $\theta$. Equation (4.1) gives a set of linear homogenous equations with unknown coefficients $\left\{\tilde{U}, \widetilde{W}, \widetilde{M}_{s}, \widetilde{N}_{s}\right\}_{(0)}^{T}$ and $\left\{\tilde{V}, \widetilde{\psi}_{s}, \widetilde{S}_{s}, \widetilde{N}_{s x}\right\}_{(0)}^{T}$, respectively, at $\theta=0$. For the existence of a nontrivial solution of these coefficients, the determinant of the coefficient matrix should be vanished. The standard procedures cannot be employed for obtaining the eigenvalues of the load factor. The nontrivial solution is found by searching the values $\bar{p}$ which make the determinant zero by using Lagrange interpolation procedure. The critical buckling load of the shell will be the smallest member of this set of values. The buckling deformations (circumferential buckling displacement mode) at any point of the cross section of the shell, for each axial half mode $m$, are determined by calculating the eigenvectors corresponding to the eigenvalues $\bar{p}$ by using Gaussian elimination procedure.

\section{Computed Results and Discussion}

A computer program based on the analysis described herein has been developed to study the buckling behaviour of the shell under consideration. The critical buckling loads and the corresponding buckling deformations of the shell are calculated numerically, and some of the results shown next are for cases that have not as yet been considered in the literature. Our study is divided into two parts in which the Poisson's ratio $v$ takes the value 0.3.

\subsection{Buckling Results}

Consider the buckling of a four-lobed cross section cylindrical shell with circumferential variable thickness under non-uniform axial loads $p(\theta)$, varying over the length $L_{2}$. The study of shell buckling is determined by finding the load factor $\bar{p}$ which equals the eigenvalues of (4.1) for each value of $m$, separately. To obtain the buckling loads $p_{B}(=\bar{p})$ we will search the set of all eigenvalues, and to obtain the critical buckling loads $p_{C}\left(<p_{B}\right)$, which correspond to loss of stability of the shell, we will search the lowest values of this set. The numerical results presented herein pertain to the buckling loads in the case of uniform and non-uniform loads for symmetric and antisymmetric type-modes.

The effect of variation in thickness on the buckling loads is presented in Table 1 which gives the fundamental buckling loads factor of a four-lobed cross section cylindrical shell with radius ratio $\zeta=0.5$ versus the axial half-wave number $m$ for the specific values of thickness ratio $\eta$, symmetric and antisymmetric type-modes. A-columns and B-columns correspond to applied non-uniform and uniform axial loads, respectively.

The results presented in this table show that the increase of the thickness ratio tends to increase the critical buckling load (bold number) for each value of $m$. These results confirm the fact that the effect of increasing the shell flexural rigidity becomes larger than that of increasing the shell mass when the thickness ratio increases. The buckling loads for antisymmetrical mode have the highest critical loads. The effect of the non-uniformity loads makes the shell has critical loads some 2-3 times lower than applied uniform loads, so that the shell buckles more readily and will be less stable for non-uniform loads. The ratio of critical loads $\mu$ takes the values within the $(1.1 \sim 2.9)$ range and takes the smallest value 1.1 for the antisymmetrical mode of the shell of constant thickness, and for the shell of variable thickness, the ratio has the biggest value 2.9. For symmetric modes, the critical buckling loads $p_{C}$ occurred with $m=5$, except for applied axial load with constant thickness which 


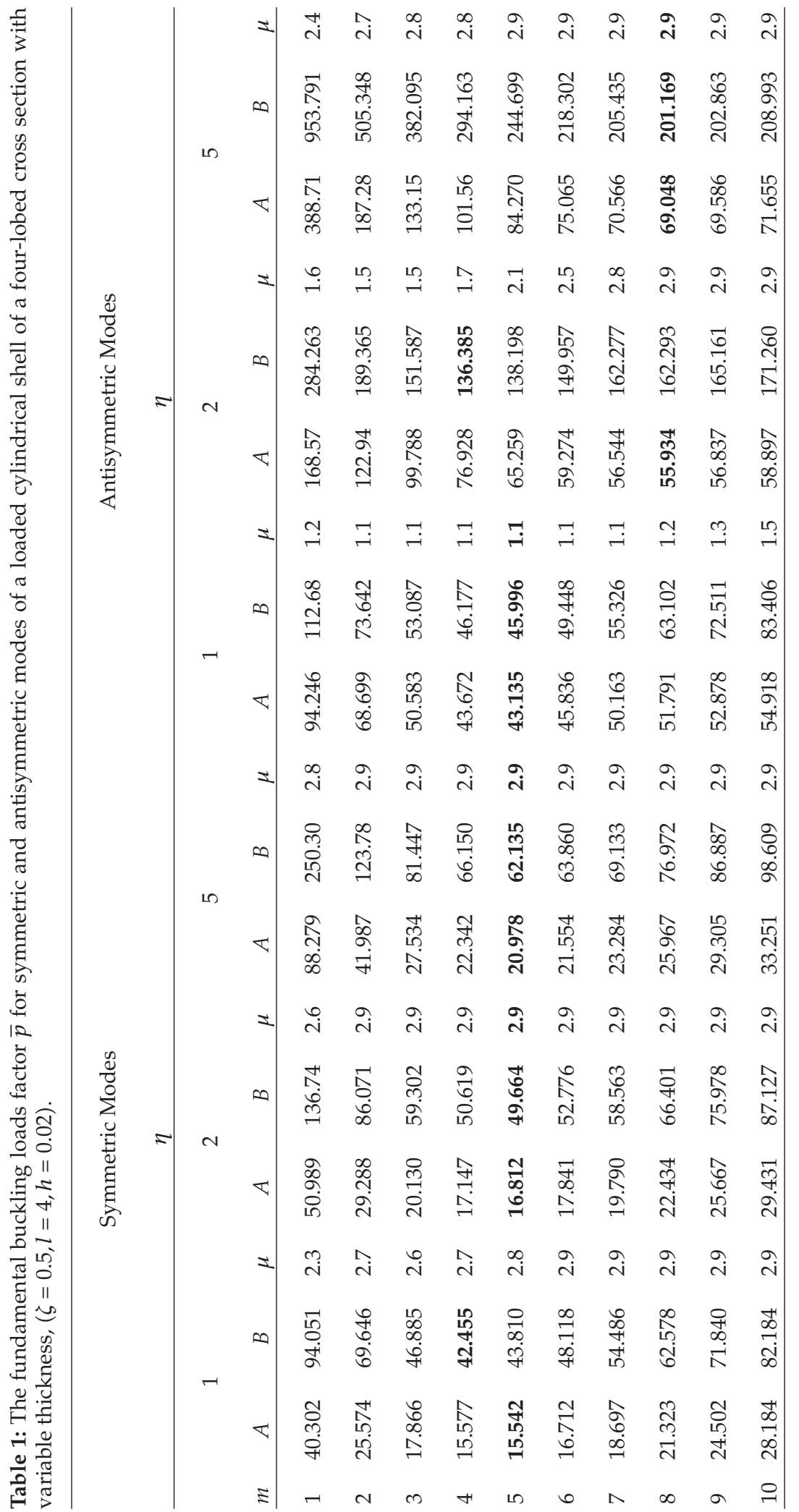


occurred with $m=4$, and for antisymmetrical modes those occurred with different values of $m(\geq 4)$ and all for $l=4$.

\subsection{Buckling Deformations}

When a structure subjected usually to compression undergoes visibly large displacement transverse to the load then it is said to buckle, and for small loads the buckle is elastic since buckling displacements disappear when the loads are removed. Generally, the buckling displacements mode is located at the weakest generatrix of the shell where the unsteady axial compression is a maximum, and the shell has less stiffness. Figures 2 and 3 show the fundamental circumferential buckling modes of a four-lobed cross section cylindrical shell of variable thickness under uniform and non-uniform loads corresponding to the critical and the buckling loads factor listed in Table 1, symmetric and antisymmetric type-modes. The thick lines show the composition of the circumferential and transverse deflections on the shell surface while the dotted lines show the original shell shape before buckling case. The numbers in the parentheses are the axial half-wave number corresponding to the critical or buckling loads. There are considerable differences between the modes of $\eta=1$ and $\eta>1$ for the symmetric and antisymmetric types of buckling deformations. For $\eta=1$, in the case of uniform axial load, the buckling modes are distributed regularly over the shell surface, but for $\eta>1$, the majority of symmetrical and antisymmetrical buckling modes, the displacements at the thinner edge are larger than those at the thicker edge, that is, the buckling modes are localized near the weakest lines on the shell surface. For $\eta=1$, in the case of non-uniform loads, the buckling modes are located at the weakest generatrix of the shell, where the axial compression load is a maximum in the compressive zone. For $\eta>1$, the modes of buckling load are concentrated near the weakest generatrix on the shell surface in the compressive zone, but the modes of critical load are located at the tensile zone, where the axial load is a minimum and the thickness is a maximum. This indicates the possibility of a static loss of stability for the shell at values of $p_{B}$ less than the critical value $p_{C}$. It can be also opined from these figures that the buckling behavior for the symmetric pattern is qualitatively similar to that of antisymmetric mode. Also, it is seen that the mode shapes are similar in the sets of the buckling modes having the ratio $\eta>2$ for the applied specific loads.

\subsection{Particular Case}

We consider a special case for a circular cylindrical shell $(\zeta=1, \eta \geq 1)$. Table 2 gives the fundamental buckling loads factor for a circular cylindrical shell of variable thickness versus the axial half-wave number under the specific load. As was expected, the symmetric and antisymmetric type-modes give the same values of buckling loads factor versus the thickness ratio. It is seen from this table, in the case of applied non-uniform axial loads, the shell will buckle more easily with increasing of axial half-wave number $m$ because the increasing of $m$ results in the decreasing of $\bar{p}$, whereas for more values of $m$ the shell is less stable. In the case of applied uniform axial loads and constant thickness $(\eta=1)$, the critical buckling load occurred for $m=1$, and an increase of $m$ results in an increase of load factor and the shell will buckle hardly for $m>1$. For $m>10$ the shell will be more stable because the values of buckling load factor increase slightly until reaching their convergence values between $(290$ 291). Whereas in the case of non-uniform axial loads a very fast convergence is observed in the lowest critical load value for $m \geq 33$. With an increase of thickness ratio $\eta$ the buckling 
A

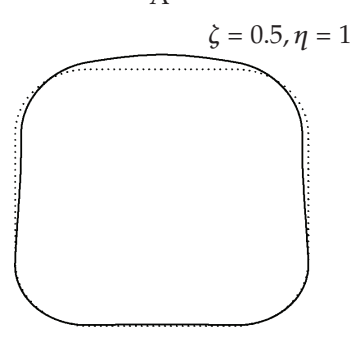

$P_{B}=40.302(1)$

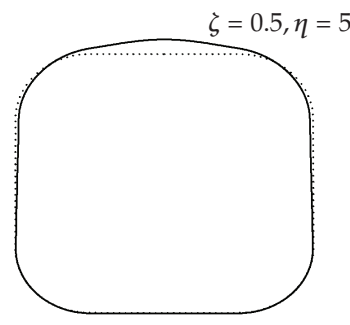

$P_{B}=88.279(1)$



$P_{C}=16.812(5)$

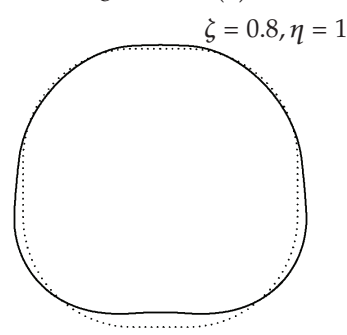

$P_{B}=103.703(1)$

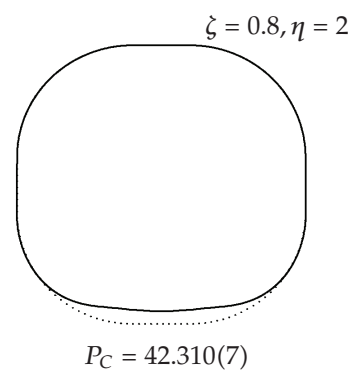

B

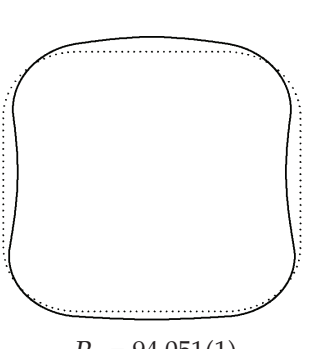

$P_{B}=94.051(1)$



$P_{B}=250.304(1)$

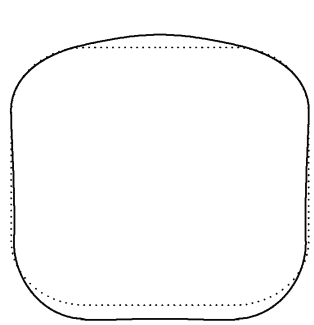

$P_{C}=49.664(5)$

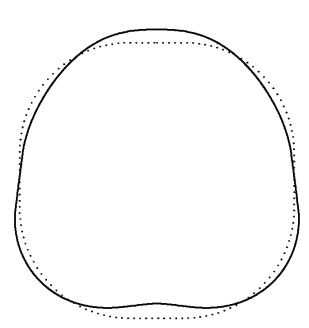

$P_{B}=226.304(1)$

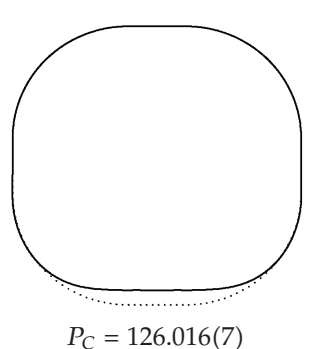

A


$P_{C}=20.978(5)$

$P_{C}=62.135(5)$

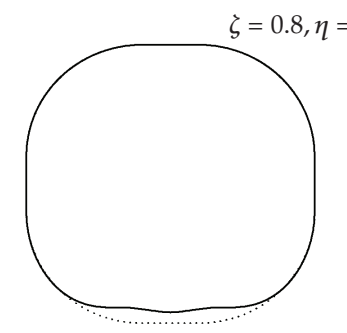

$P_{C}=40.985(7)$
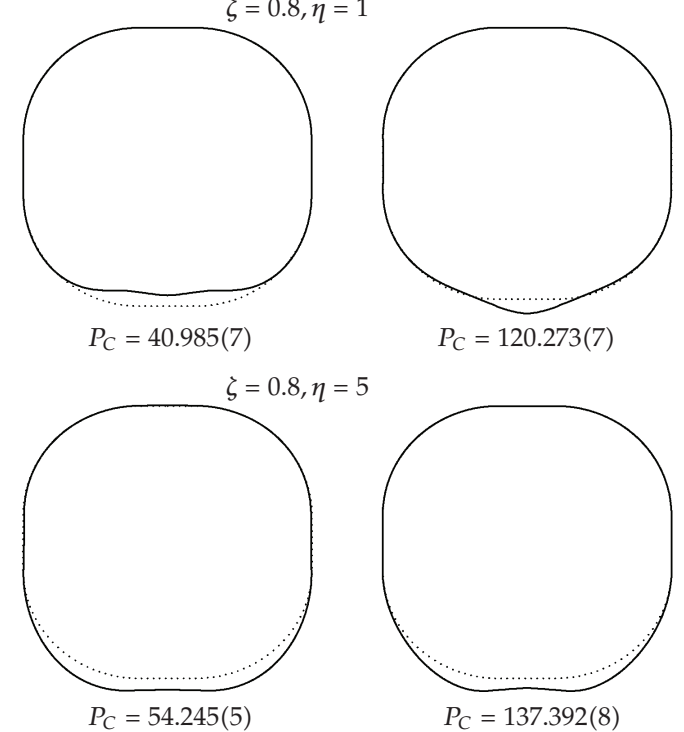

Figure 2: The symmetric buckling deformations of a cylindrical shell of a four-lobed cross section with variable thickness. $\{l=4, h=0.02\}$. 
A
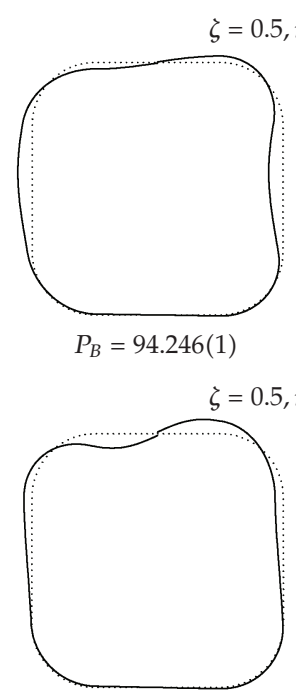

$P_{B}=388.71(1)$

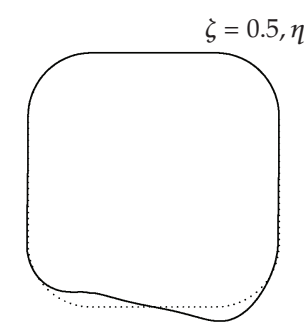

$P_{\mathrm{C}}=55.934(8)$

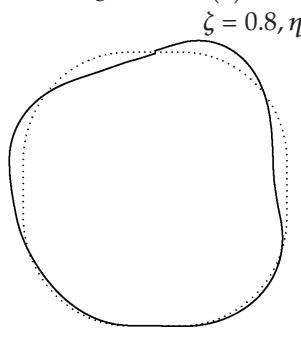

$P_{B}=146.421(1)$

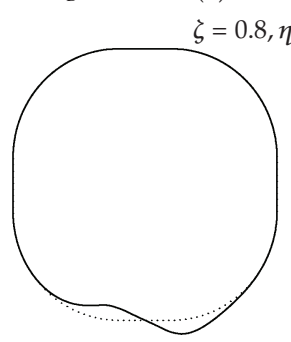

$P_{C}=114.942(12)$
B

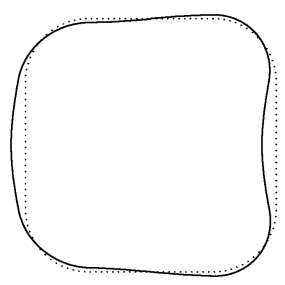

$P_{B}=112.686(1)$



$P_{B}=953.791(1)$

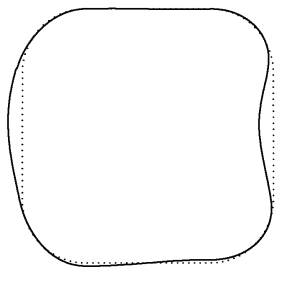

$P_{C}=136.385(4)$

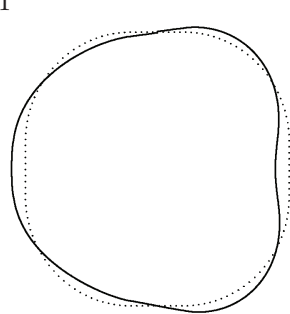

$P_{B}=226.361(1)$

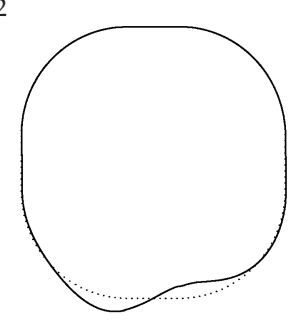

$P_{C}=320.546(6)$
A

B

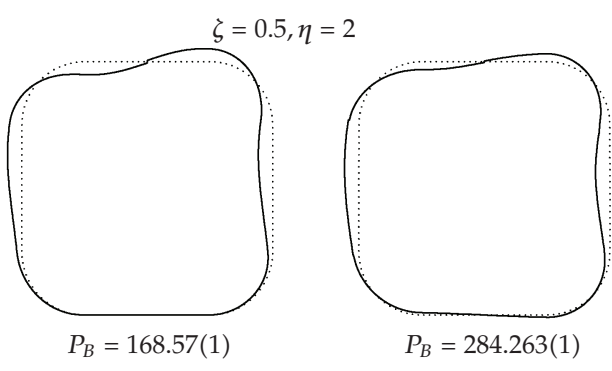

$\zeta=0.5, \eta=1$
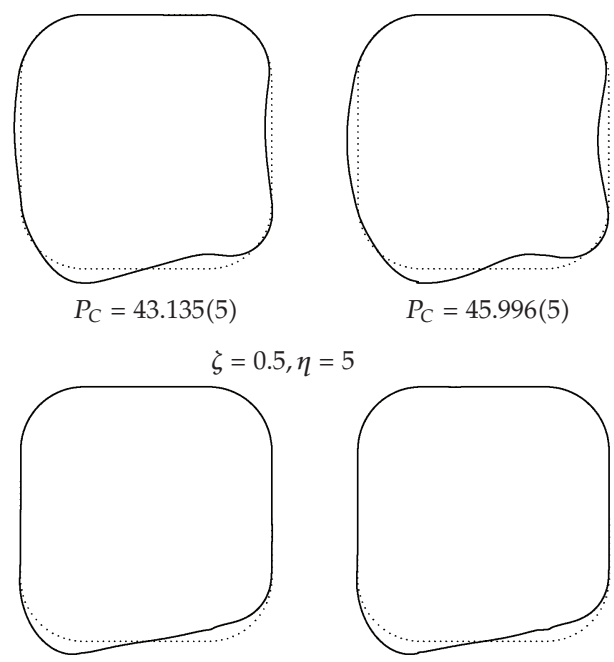

$P_{C}=69.048(8)$

$\zeta=0.8, \eta=1$

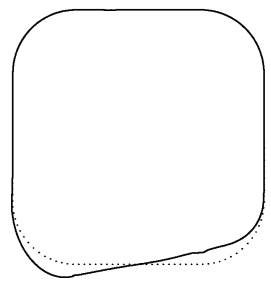

$P_{C}=201.169(8)$
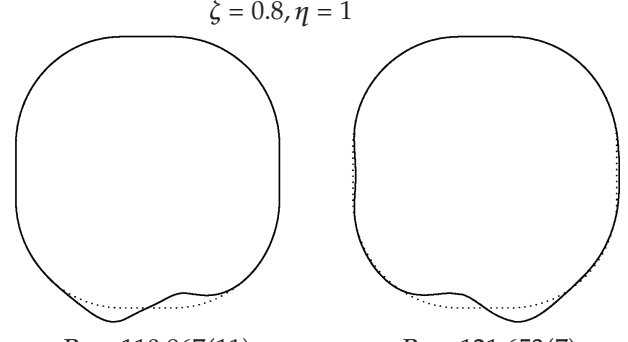

$P_{C}=110.867(11)$

$P_{C}=121.653(7)$

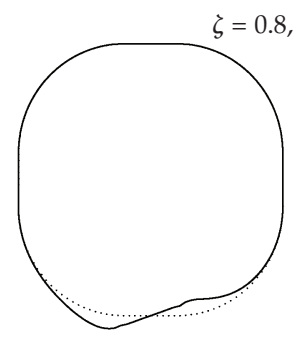

$P_{C}=126.578(12)$

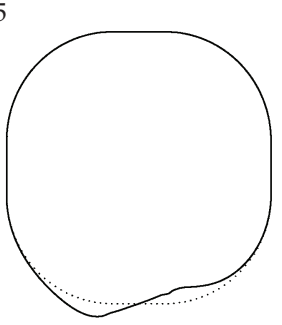

$P_{C}=374.472(12)$

Figure 3: The antisymmetric buckling deformations of a cylindrical shell of a four-lobed cross section with variable thickness. $\{l=4, h=0.02\}$. 
Table 2: The fundamental buckling loads factor $\bar{p}$ for symmetric and antisymmetric modes of an axially loaded cylindrical shell, $(\zeta=1, l=4, h=0.02)$.

\begin{tabular}{|c|c|c|c|c|c|c|c|c|c|}
\hline \multicolumn{10}{|c|}{ Symmetric \& Antisymmetric Modes } \\
\hline \multirow[b]{3}{*}{$m$} & \multirow{2}{*}{\multicolumn{2}{|c|}{1}} & \multirow{2}{*}{\multicolumn{4}{|c|}{$\eta$}} & & & \\
\hline & & & & & & & \multicolumn{2}{|c|}{5} & \\
\hline & A & $B$ & $\mu$ & A & $B$ & $\mu$ & A & $B$ & $\mu$ \\
\hline 1 & 143.814 & 267.355 & 1.8 & 189.854 & 457.252 & 2.4 & 335.448 & 894.988 & 2.6 \\
\hline 2 & 135.864 & 295.683 & 2.1 & 164.134 & 432.286 & 2.6 & 241.186 & 673.852 & 2.7 \\
\hline 3 & 130.580 & 301.418 & 2.3 & 151.581 & 411.782 & 2.7 & 204.525 & 581.959 & 2.8 \\
\hline 4 & 127.165 & 404.754 & 3.1 & 143.553 & 396.756 & 2.7 & 183.601 & 527.618 & 2.8 \\
\hline 5 & 124.319 & 309.743 & 2.5 & 137.783 & 385.323 & 2.7 & 169.798 & 491.308 & 2.8 \\
\hline 6 & 122.243 & 312.076 & 2.5 & 133.457 & 376.613 & 2.8 & 159.812 & 464.874 & 2.9 \\
\hline 7 & 120.566 & 315.157 & 2.6 & 130.102 & 369.922 & 2.8 & 152.083 & 444.416 & 2.9 \\
\hline 8 & 119.173 & 314.672 & 2.6 & 127.390 & 364.647 & 2.8 & 145.726 & 427.695 & 2.9 \\
\hline 9 & 117.985 & 312.156 & 2.6 & 125.647 & 360.347 & 2.8 & 140.133 & 413.152 & 2.9 \\
\hline 10 & 116.947 & 293.795 & 2.5 & 123.156 & 356.685 & 2.8 & 134.853 & 399.486 & 2.9 \\
\hline
\end{tabular}

A

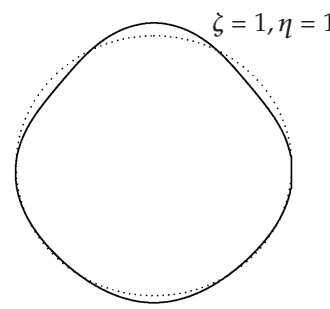

$P_{B}=143.814(1)$

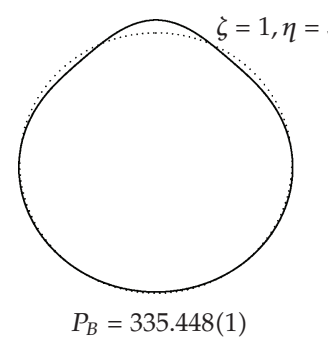

B

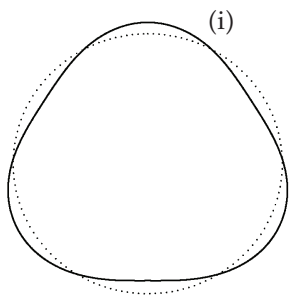

$P_{C}=267.355(1)$

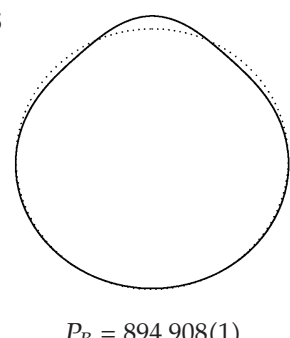

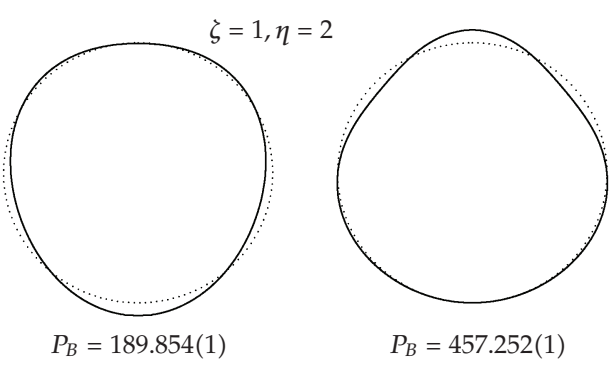

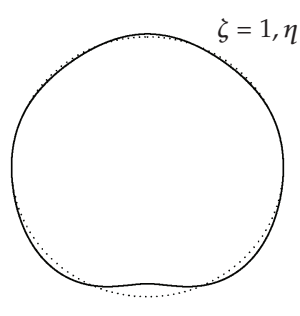

$P_{B}=135.864(2)$

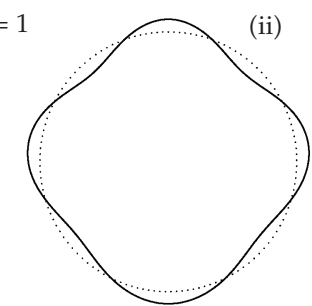

$P_{B}=295.683(2)$

Figure 4: The circumferential buckling modes of a circular cylindrical shell with variable thickness.

loads increase for the uniform and non-uniform loads, and they are lower values for the shell when the non-uniform loads applied. For $\eta>1$, the ratio of critical loads $\mu$ is nearly equal to 2.9 .

Figure 4 shows the circumferential buckling modes of a circular cylindrical shell with variable thickness under the specific load. It is seen from this figure that the buckling deformations for applied uniform loads are distributed regularly over the shell surface of constant thickness, see (i), (ii) in Figure 4. These figures are in quite good agreement with 


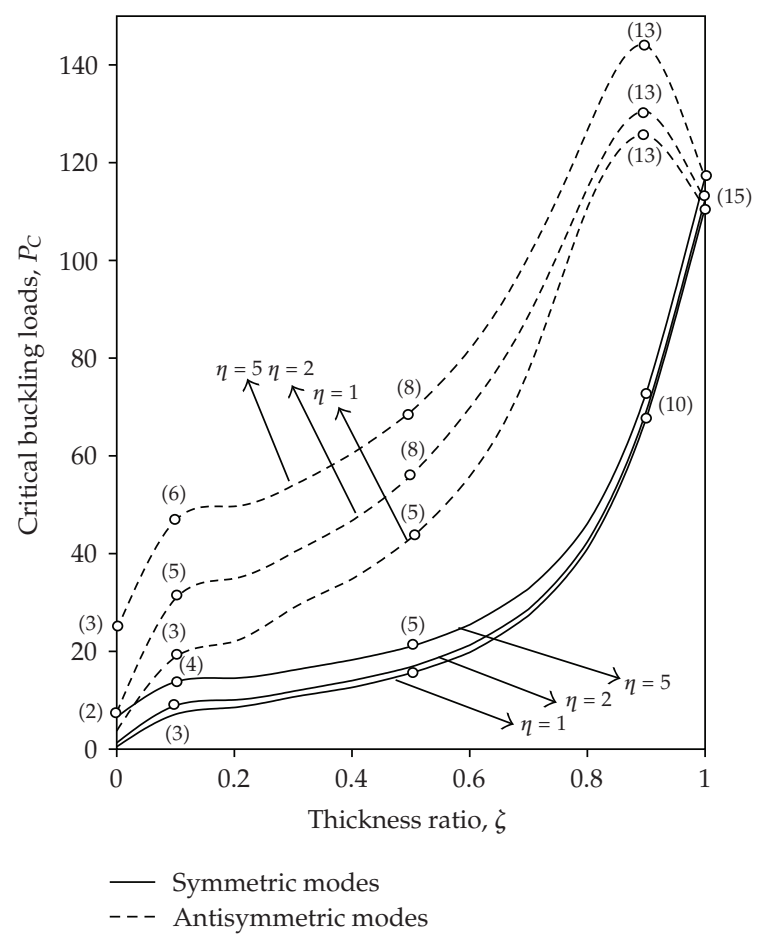

Figure 5: Critical buckling loads versus thickness ratio of a four-lobed cross section cylindrical shell with variable thickness, $(l=4, h=0.02)$.

[5]. It can also be seen from this figure that the shell of applied non-uniform loads buckles more easily than one of applied uniform loads.

Figure 5 shows the variations in the critical buckling loads of a non-uniformly loaded shell of a four-lobed cross section versus the radius ration $\zeta$, for the specific values of thickness ratio $\eta$. The axial half-wave number of corresponding critical buckling loads is shown in this figure as $(m)$. It is seen from this figure, for the symmetric and antisymmetric type-modes, that an increase in the radius ratio $\zeta$ causes an increase in the critical loads $p_{C}$, and when the foregoing ratio becomes unity the latter quantities take the same values and are assumed to be for a circular cylindrical shell. It is observed that the critical loads increase with an increase in the thickness ratio where the shell becomes more stiffness. Upon increasing the radius ratio, the critical buckling axial half-wave number increases. The nominal axial halfwave number corresponding to the critical buckling load may be in general depends on the radius of curvature at the lobed corners of the shell.

\section{Conclusions}

An approximate analysis for studying the elastic buckling characteristics of circumferentially non-uniformly axially loaded cylindrical shell of a four-lobed cross section having circumferential varying thickness is presented. The computed results presented herein pertain to the buckling loads and the corresponding mode shapes of buckling displacements by using the transfer matrix approach. The method is based on thin-shell theory and applied to a shell 
of symmetric and antisymmetric type-modes, and the analytic solutions are formulated to overcome the mathematical difficulties associated with mode coupling caused by variable shell wall curvature and thickness. The fundamental buckling loads and corresponding buckling deformations have been presented, and the effects of the thickness ratio of the crosssection and the non-uniformity of applied load on the critical loads and buckling modes were examined.

The study showed that the buckling strength for non-uniform loads was lower than that under uniform axial loads. The deformation of corresponding buckling load are located at the compressive zone of a small thickness but, in contrast, the deformation of corresponding critical load are located at the tensile zone of a large thickness, and this indicates the possibility of a static loss of stability for the shell at values of $p_{B}$ less than the critical value $p_{C}$. Generally, the symmetric and antisymmetric buckling deformations take place in the less stiffened zones of the shell surface where the lobes are located. However, for the applied uniform and non-uniform axial loads, the critical buckling loads increase with either increasing radius ratio or increasing thickness ratio and become larger for a circular cylindrical shell.

\section{Acknowledgment}

The author is grateful to anonymous reviewers for their good efforts and valuable comments which helped to improve the quality of this paper.

\section{References}

[1] P. Seide and V. I. Weingarten, "On the buckling of circular cylindrical shells under pure bending," Journal of Applied Mechanics, vol. 28, pp. 112-116, 1961.

[2] G. Gerard, "Compressive stability of orthotropic cylinders," Journal of Aerospace Science, vol. 29, pp. $1171-1179,1962$.

[3] N. J. Hoff and T.-C. Soong, "Buckling of circular cylindrical shells in axial compression," International Journal of Mechanical Sciences, vol. 7, no. 7, pp. 489-520, 1965.

[4] Y. Stavsky and S. Friedland, "Stability of heterogeneous orthotropic cylindrical shells in axial compression," Israel Journal of Technology, vol. 7, pp. 111-119, 1969.

[5] G. Yamada, T. Irie, and M. Tsushima, "Vibration and stability of orthotropic circular cylindrical shells subjected to axial load," Journal of the Acoustical Society of America, vol. 75, no. 3, pp. 842-848, 1984.

[6] M. Sabag, Y. Stavsky, and J. B. Greenberg, "Buckling of edge damaged, cylindrical composite shells," Journal of Applied Mechanics, vol. 56, no. 1, pp. 121-126, 1989.

[7] W. T. Koiter, I. Elishakoff, Y. W. Li, and J. H. Starnes Jr., "Buckling of an axially compressed cylindrical shell of variable thickness," International Journal of Solids and Structures, vol. 31, no. 6, pp. 797-805, 1994.

[8] J. G. Teng, "Buckling of thin shells: recent advances and trends," Applied Mechanics Reviews, vol. 49, no. 4, pp. 263-274, 1996.

[9] J. B. Greenberg and Y. Stavsky, "Vibrations and buckling of composite orthotropic cylindrical shells with nonuniform axial loads," Composites Part B, vol. 29, no. 6, pp. 695-703, 1998.

[10] A. E. Love, A Treatise on the Mathematical Theory of Elasticity, Dover, New York, NY, USA, 1944.

[11] W. Flügge, Stresses in Shells, Springer, Berlin, Germany, 1973.

[12] P. E. Tovstik, Stability of Thin Shells, Nauka, Moscow, Russia, 1995.

[13] W. Flügge, "Die Stabilitat der Kreiszylinderschale," Ingenieur-Archiv, vol. 3, no. 5, pp. 463-506, 1932.

[14] P. P. Bijlaard and R. H. Gallagher, "Elastic instability of a cylindrical shell under arbitrary circumferential variation of axial stress," Journal of the Aerospace Sciences, vol. 27, no. 11, pp. 854-858, 1960.

[15] J. B. Greenberg and Y. Stavsky, "Buckling of composite orthotropic cylindrical shells under nonuniform axial loads," Composite Structures, vol. 30, no. 4, pp. 399-406, 1995. 
[16] J. B. Greenberg and Y. Stavsky, "Vibrations and buckling of composite orthotropic cylindrical shells with nonuniform axial loads," Composites Part B, vol. 29, no. 6, pp. 695-703, 1998.

[17] I. V. Avdoshka and G. I. Mikhasev, "Wave packets in a thin cylindrical shell under a non-uniform axial load," Journal of Applied Mathematics and Mechanics, vol. 65, no. 2, pp. 301-309, 2001.

[18] C. Song, "Buckling of un-stiffened cylindrical shell under non-uniform axial compressive stress," Journal of Zhejiang University Science, vol. 3, no. 5, pp. 520-531, 2002.

[19] A. H. Sofiyev and H. Erdem, "The stability of non-homogeneous elastic cylindrical thin shells with variable thickness under a dynamic external pressure," Turkish Journal of Engineering and Environmental Sciences, vol. 26, no. 2, pp. 155-166, 2002.

[20] L. S. Eliseeva and S. B. Filippov, "Buckling and vibrations of a cylindrical shell of variable thickness with a curvilinear edge," Vestnik Sankt-Peterburgskogo Universiteta, no. 3, pp. 84-91, 2003.

[21] S. B. Filippov, D. N. Ivanov, and N. V. Naumova, "Free vibrations and buckling of a thin cylindrical shell of variable thickness with curve linear edge," Technische Mechanik, vol. 25, no. 1, pp. 1-8, 2005.

[22] W. H. Duan and C. G. Koh, "Axisymmetric transverse vibrations of circular cylindrical shells with variable thickness," Journal of Sound and Vibration, vol. 317, no. 3-5, pp. 1035-1041, 2008.

[23] C. T. Sambandam, B. P. Patel, S. S. Gupta, C. S. Munot, and M. Ganapathi, "Buckling characteristics of cross-ply elliptical cylinders under axial compression," Composite Structures, vol. 62, no. 1, pp. 7-17, 2003.

[24] N. Silvestre, "Buckling behaviour of elliptical cylindrical shells and tubes under compression," International Journal of Solids and Structures, vol. 45, no. 16, pp. 4427-4447, 2008.

[25] A. Tesar and L. Fillo, Transfer Matrix Method, vol. 35 of Mathematics and Its Applications (East European Series), Kluwer Academic Publishers, Dordrecht, The Netherlands, 1988.

[26] A. L. Goldenveizer, Theory of Elastic Thin Shells, International Series of Monographs on Aeronautics and Astronautics, Pergamon Press, Oxford, UK, 1961.

[27] V. V. Novozhilov, Thin Shell Theory, Second Augmented and Revised Edition, P. Noordhoff, Groningen, The Netherlands, 1964.

[28] R. Uhrig, Elastostatikund Elastokinetikin Matrizenschreibweise, Springer, Berlin, Germany, 1973. 


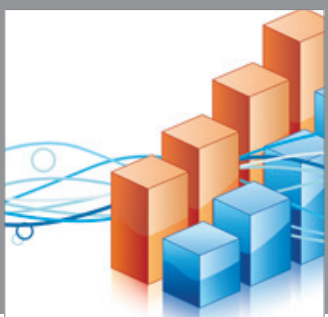

Advances in

Operations Research

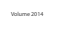

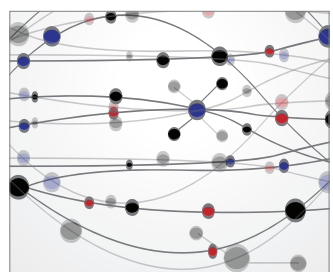

\section{The Scientific} World Journal
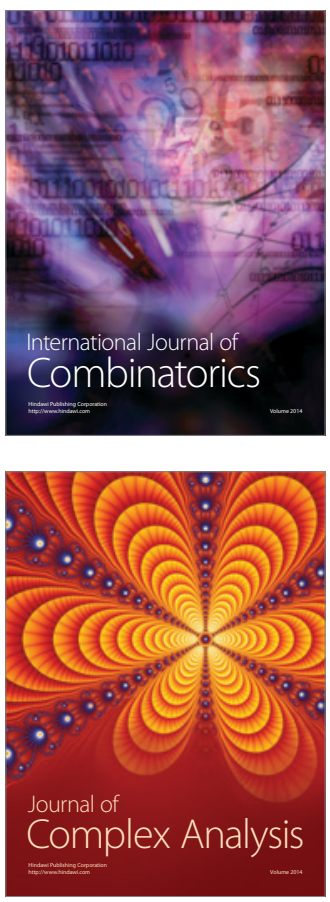

International Journal of

Mathematics and

Mathematical

Sciences
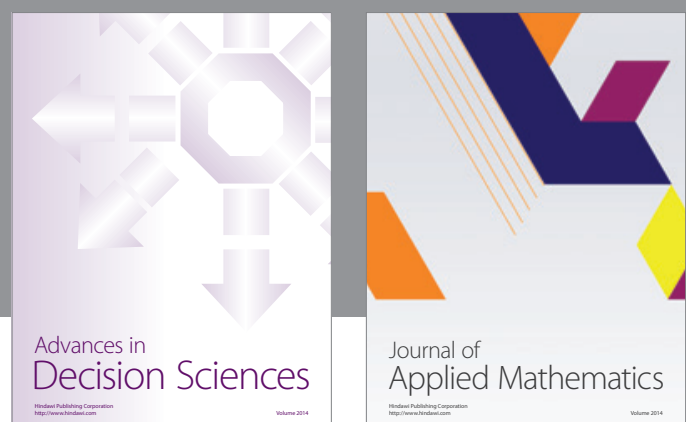

Journal of

Applied Mathematics


Submit your manuscripts at http://www.hindawi.com
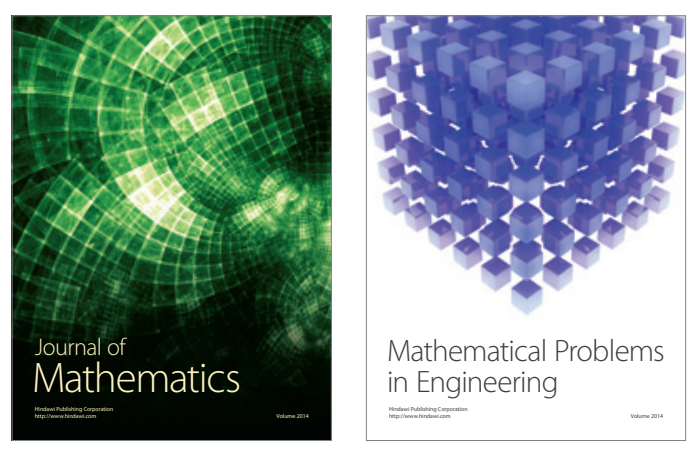

Mathematical Problems in Engineering
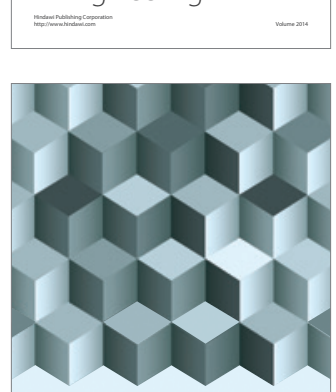

Journal of

Function Spaces
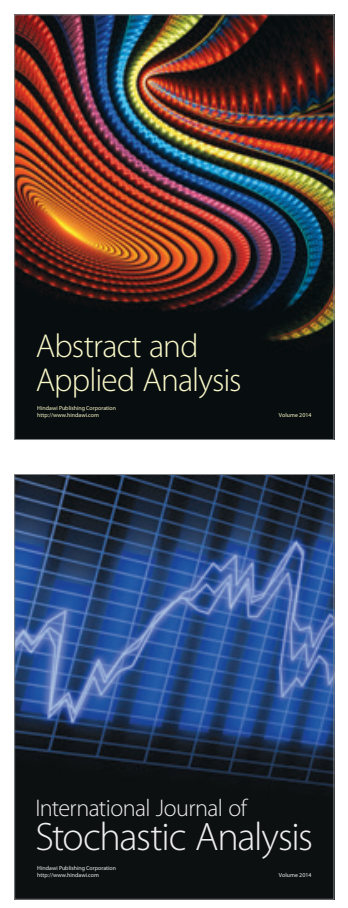



ournal of

Probability and Statistics

Promensencen
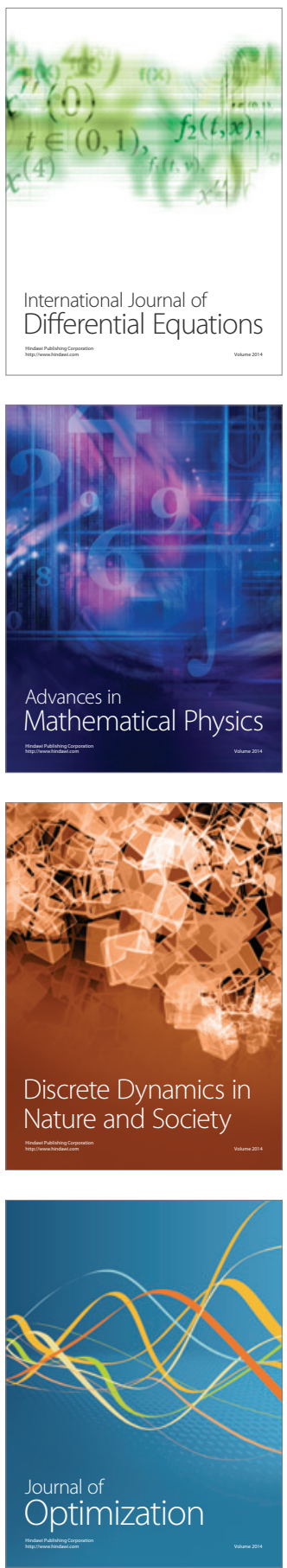